\title{
FATORES ANTINUTRICIONAIS: INIBIDORES DE PROTEASES E LECTINAS
}

\author{
ANTINUTRITIONAL FACTORS: PROTEASE INHIBITORS AND LECTINS
}

Mara Reis SILVA'

Maria Aparecida Azevedo Pereira da SILVA²

\begin{abstract}
RESUMO
Os fatores antinutricionais presentes em alimentos podem provocar efeitos fisiológicos adversos ou diminuir a biodisponibilidade de nutrientes. A maior questão sobre os riscos à saúde provocados por antinutrientes é o desconhecimento dos níveis de tolerância, do grau de variação do risco individual e da influência de fatores ambientais sobre a capacidade de detoxificação do organismo humano. Dentre os fatores antinutricionais os inibidores de proteases e as lectinas são considerados instáveis ao tratamento térmico. A hipertrofia pancreática causada pelos inibidores de tripsina tem sido relatada em alguns estudos com animais. As alterações da função fisiológica em animais causadas por ação de lectinas no intestino parecem estar relacionadas à especificidade destas substâncias com as células da mucosa intestinal. Os possíveis efeitos adversos dos inibidores de proteases e das lectinas na maioria das vezes são inferidos somente de experimentos com animais de laboratório.
\end{abstract}

Termos de indexação: fatores antinutricionais, inibidores da protease, inibidores da tripsina, fosfatidilcolinas.

\begin{abstract}
The antinutritional factors present in foods can cause adverse physiological effects or decrease the bioavailability of nutrients. Health risk factors associated with antinutrients include: lack of knowledge of the tolerance levels to these compounds in the human organism, little available information on the degree of variation of individual risks and little knowledge with respect to the influence of environmental factors on the detoxification capacity of the human organism. The possible adverse effects of protease inhibitors and lectin on human health are, in most cases, only inferred by way of experiments with laboratory animals. Pancreatic hypertrophy caused by trypsin inhibitors has been shown in some animal experiments. Alterations in physiological functions at the intestinal level shown by animals submitted to lectins containing diets seem to be related to the specificity of these substances with the intestinal mucosa cells. There is no evidence that the trypsin inhibitors and lectins present in adequately processed foods are toxic to humans.
\end{abstract}

Index terms: antinutritional factors, protease inhibitors, trypsin inhibitors, phosphatidylcholines.

\section{INIBIDORES DE PROTEASES}

Os inibidores de proteases são proteínas de ampla distribuição no reino vegetal, capazes de inibir as atividades da tripsina, quimotripsina, amilase e carboxipeptidase (Bender, 1987; Xavier-Filho \& Campos, 1989). Geralmente, são denominados como inibidores da primeira enzima contra a qual foram testados e na maioria das

(1) Faculdade de Nutrição, Universidade Federal de Goiás, Caixa Postal 131, 74605-080, Goiânia, GO, Brasil, Fax (0xx62) 202-1748, Fone (0xx62) 202-3549. Correspondência para/Correspondence to: M. R. SILVA.

(2) Departamento de Planejamento Alimentar e Nutrição, Faculdade de Engenharia de Alimentos, Universidade Estadual de Campinas, Caixa Postal 6121, 13083-970, Campinas, SP, Brasil. 
pesquisas foi investigada a tripsina (Sgarbieri \& Whitaker, 1982).

A pesquisa de inibidores de proteases foi centrada, principalmente, nos inibidores de tripsina encontrados nas sementes de leguminosas, mais especificamente na soja, os quais foram supostamente responsabilizados pelo baixo valor nutritivo de leguminosas cruas (Xavier-Filho \& Campos, 1989).

De acordo com Proll et al. (1998) as leguminosas de maneira geral podem conter fatores antinutricionais e outras substâncias nocivas a saúde, desta forma, grãos não convencionais com potencial de uso na alimentação, devem ser testados em dietas animais antes da utilização em dietas humanas.

\section{Características químicas e físicas}

Os inibidores de proteases da soja são classificados em duas principais categorias: os de alto peso molecular, com aproximadamente 20.000, que apresentam duas pontes dissulfeto, 181 resíduos de aminoácidos e possuem especificidade primária para tripsina e os de peso molecular entre 6.000 e 10.000, com alta proporção de ligações dissulfeto, 71 resíduos de aminoácidos e capacidade para inibir tripsina e quimotripsina em sítios de ligação independentes. Estes inibidores são referidos como tipo Kunitz e Bowman-Birk, respectivamente (Liener, 1994).

Os inibidores tipo Kunitz são encontrados em todas as subfamílias Leguminosae: Mimosoideae, Caesalpinoideae, Papilionoideae e também na família Solanaceae (Batista et al., 1996).

Koide \& Ikenaka (1973) estabeleceram a seqüência completa de aminoácidos do inibidor de Kunitz da soja. A seqüência completa de aminoácidos para isoinibidores de tripsina já foi obtida de um número considerável de inibidores típicos de sementes de leguminosas, os quais foram adicionados às classes dos inibidores de Kunitz e Bowman-Birk (Xavier-Filho \& Campos, 1989).

A estabilidade térmica dos inibidores de proteases depende de seu peso molecular e do grau de estabilização da conformação ativa por pontes de dissulfeto (Belitz \& Grosch, 1988). O inibidor de Bowman-Birk da soja é mais estável ao calor e às variações do $\mathrm{pH}$ do que o inibidor de Kunitz, devido às diferenças de tamanho e número de ligações dissulfeto (Sgarbieri \& Whitaker, 1982).

Antunes \& Sgarbieri (1980) obtiveram inativação total de inibidor de tripsina em feijões (Phaseolus vulgaris) embebidos em água destilada por uma noite e submetidos a temperatura de $97^{\circ} \mathrm{C}$ por 7,5 minutos. Esse fato, demonstra, segundo os autores, que inativação total do inibidor de tripsina pode ser alcançada em feijões embebidos em água e aquecidos a $100^{\circ} \mathrm{C}$ por 5 a 10 minutos.

Mulimani \& Paramjyothi (1993) observaram redução da atividade de inibidor de tripsina e quimotripsina em feijão-guandu (Cajanus cajan L.) através de aplicação de radiação ultravioleta por 90 minutos e aquecimento dos grãos, previamente embebidos em água, a $100^{\circ} \mathrm{C}$ por 5 minutos.

A facilidade relativa com que os inibidores de proteases podem ser destruídos pelo calor permitiu o uso popular de feijões, como uma fonte importante de proteína na dieta humana e animal (Liener, 1994).

\section{Atividade biológica}

A descoberta dos inibidores de proteases provenientes de leguminosas, particularmente da soja, estimularam pesquisas sobre a ação em animais experimentais, devido a sua interferência na nutrição animal (Rackis, 1974).

Os efeitos nocivos dos inibidores de proteases em animais alimentados com leguminosa crua são complexos. Muitos estudos com animais monogástricos têm atribuído aos efeitos deletérios, principalmente alterações metabólicas do pâncreas (aumento da secreção enzimática, hipertrofia e hiperplasia) e redução da taxa de crescimento, à presença de inibidores de tripsina na alimentação à base de leguminosas (Al-Wesali et al., 1995).

Nitsan \& Liener (1976) estudaram o efeito de dietas com farinha de soja crua e aquecida sobre os níveis de tripsina, quimotripsina e amilase no pâncreas de ratos. Os autores concluíram que a ingestão de soja crua, ao contrário da soja cozida, estimulou a secreção das enzimas pancreáticas.

Churella et al. (1976) não observaram hipertrofia ou hiperplasia pancreática em ratos alimentados com fórmulas infantis à base de soja. Os autores sugerem que o tratamento térmico das fórmulas pesquisadas foi suficiente para reduzir a atividade do inibidor de tripsina a baixos níveis, portanto, sem qualquer significância nutricional.

Abbey et al. (1979b) relataram a possível existência de outros fatores além do inibidor de tripsina em feijão cru, capazes de causarem hipertrofia pancreática e elevação dos níveis de tripsina e quimotripsina no intestino de ratos.

As evidências experimentais induzem a aceitação do mecanismo de inibição retroativa do controle da secreção do pâncreas, para a explicação da hipertrofia pancreática provocada em ratos com administração de altas doses de inibidor de tripsina. No mecanismo de inibição retroativa proposto para a regulação da secreção enzimática do pâncreas, os níveis de tripsina e/ou quimotripsina livres no intestino delgado determinam a quantidade de secreção pancreática, isto é, quando o nível de tripsina abaixa a um certo limiar o pâncreas é induzido através da colecistoquinina a secretar mais enzima (Rackis \& Gumbmann, 1982). O inibidor de tripsina bloqueia a ação da tripsina resultando em aumento excessivo da concentração plasmática de colecistoquinina, 
e desta forma, o pâncreas é continuamente estimulado a liberar mais enzima, provocando hipertrofia pancreática (Liddle et al., 1984).

Bender (1987) relata que existem discordâncias entre autores sobre a hipertrofia de pâncreas em animais. Uma vez que, alguns pesquisadores observaram aumento do número de células e outros aumento do tamanho das células ou ambos fenômenos.

Os resultados da pesquisa de Grant et al. (1988) com ratos alimentados com proteína de soja mostraram considerável aumento do tamanho do pâncreas dos animais. Diferentemente de ratos e galinhas, a ingestão de farinha de soja e feijão crus por porcos da Índia, bezerros, cachorros e porcos não provoca hipertrofia pancreática, contudo, observa-se hiposecreção das enzimas pancreáticas e sérica, depressão do ganho de peso corporal ou perda de peso de animais (Hasdai et al., 1989).

A inibição do crescimento em animais jovens alimentados com leguminosas cruas é provocada pela excessiva perda fecal de proteína secretada pelo pâncreas, visto que as enzimas pancreáticas são ricas em aminoácidos sulfurados e esta perda endógena não pode ser compensada pela ingestão de proteína de leguminosas (Rackis \& Gumbmann, 1982). Liddle et al. (1984) observaram elevação dos níveis de colecistoquinina em ratos, após a aplicação intragástrica de inibidor de tripsina extraído de soja.

O inibidor de Bowman-Birk é relativamente estável à exposição do suco gástrico humano (Liener, 1986). A tripsina humana é fracamente inibida pelo inibidor de Kunitz, na base de mol/mol (Bender, 1987).

Embora a hipertrofia pancreática seja freqüentemente reportada em animais de laboratório, e supostamente desencadeada pela presença de inibidores de protease, não existem evidências de efeitos deletérios em seres humanos (Deshpande \& Nielsen, 1987). Ainda que, mesmo no caso de alguns grãos consumidos crus, como o amendoim (Arachis hypogaea) que comprovadamente possui inibidor de tripsina e quimotripsina, não há qualquer relato de efeito nocivo a saúde (Bender, 1987).

No entanto, Liener et al. (1988) observaram um efeito estimulatório do inibidor de Bowman-Birk na atividade do pâncreas de seres humanos. Quando foi adicionado mais do que $4 \mathrm{mg}$ de inibidor por $\mathrm{ml}$ de suco pancreático houve aproximadamente $95 \%$ de inibição da tripsina e 50\% de inibição da elastase.

\section{LECTINAS}

A definição de lectina mais completa foi formulada por Kocourek \& Horejsi citados por Etzler (1985). De acordo com estes autores, lectinas são proteínas não pertencentes ao sistema imunológico, porém capazes de reconhecer sítios específicos em moléculas e ligar-se reversivelmente a carboidratos, sem alterar a estrutura covalente das ligações glicosídicas dos sítios.

A nomenclatura das lectinas é originada da denominação científica das espécies em que são purificadas, de acordo com o protocolo de purificação, pela designação dos monossacarídeos aos quais têm especificidade ou ainda pela designação do tecido ao qual foram extraídas. Assim a lectina da Vicia faba é conhecida como favina, a concanavalina A é uma lectina obtida da Canavalia ensiformis, e D-galactose-N-acetil-D-galactosamina- é uma lectina específica da Erythrina cristagalli (Kennedy et al., 1995).

\section{Propriedades biológicas}

As lectinas ou hemaglutininas podem ser caracterizadas e detectadas por sua habilidade em aglutinar eritrócitos, em certos casos com alta especificidade (Lis \& Sharon, 1973; Askar, 1986). Algumas lectinas são específicas em suas reações com grupos sangüíneos humanos $A B O$ e $M N$ e subgrupo $A_{1}$ (Sharon \& Lis, 1972). Todos estes efeitos são produzidos pela habilidade das lectinas de se ligarem a tipos específicos de açúcares na superfície celular (Deshpande \& Damodaran, 1990). Além dessas propriedades, as lectinas podem promover estimulação mitogênica de linfócitos e aglutinação de células cancerosas (Lis \& Sharon, 1973; Liener, 1981).

Embora muitas lectinas reconheçam e se liguem a açúcares simples tais como glicose, manose, galactose, $\mathrm{N}$ -acetilgalactosamina, $\mathrm{N}$-acetilglucosamina ou fucose, a afinidade é muito maior para com os constituintes de glicoproteínas: ácido siálico e $\mathrm{N}$-acetilgalactosamina contendo cadeias de glicanos, encontrados em animais e seres humanos (Nicolson, 1974; Peumans \& Van Damme, 1996).

A especificidade das lectinas com relação à diferentes carboidratos possibilita a sua utilização em pesquisas na área biológica e médica tais como: investigação da superfície de células, caracterização de eritrócitos, como agentes mitogênicos, caracterização de estádios de desenvolvimento de microorganismos diversos, purificação de glicoproteínas, morfologia de neurônios e identificação de conexões neurais no sistema nervoso central (Kennedy et al., 1995).

As lectinas são encontradas em uma ampla variedade de espécies de plantas e animais, entretanto, estas substâncias estão presentes em maior quantidade em grãos de leguminosas e gramíneas (Mancini Filho et al., 1979; Pusztai, 1989). Martin-Cabrejas et al. (1995) encontraram quantidades consideráveis de inibidores de tripsina/quimotripsina e $\alpha$-amilase e elevada atividade de lectinas em cinco cultivares de feijões (Phaseolus vulgaris) frescos e estocados por cinco anos. Muitas lectinas também 
já foram isoladas e caracterizadas de diversas variedades de cogumelos (Kawagishi et al., 1996).

Exemplos de lectinas bem caracterizadas são: concanavalina A presente em jack bean (Canavalia ensiformis), aglutinina da soja e aglutinina do gérmen de trigo (Sharon \& Lis, 1972).

O fato das lectinas terem larga distribuição em plantas sugere alguma importância fisiológica para estas substâncias (Liener, 1976; Etzler, 1985). As funções das lectinas podem ser variadas e parecem ter relação com os estádios de maturação e germinação das sementes (Howard et al., 1972), assim como parecem ter relação com os mecanismos de defesa da planta contra o ataque de fungos (Liener, 1976).

\section{Inativação das lectinas}

A inativação de lectinas ou redução da atividade hemaglutinante a valores negligenciáveis é usualmente obtida por métodos tradicionais de preparo doméstico ou processamento industrial dos alimentos (Muelenaere, 1965; Antunes \& Sgarbieri, 1977; Pak et al., 1978; Noah et al., 1980; Askar, 1986; Ojimelukwe et al., 1995).

Embora os efeitos tóxicos das lectinas de leguminosas possam geralmente ser eliminados por tratamento térmico apropriado, algumas condições, como calor seco são pouco efetivas para inativação de lectinas (Liener, 1974).

Mancini Filho et al. (1979) obtiveram inativação total de lectina em uma solução purificada contendo 475 $\mu \mathrm{g}$ de proteína $/ \mathrm{ml}$, submetida a temperatura de $90^{\circ} \mathrm{C}$ durante 5 minutos. Estes autores sugerem que a alta sensibilidade térmica das lectinas em solução pode explicar a facilidade de inativação de lectinas em grãos de feijões, quando previamente embebidos em água.

Barca et al. (1991) analisaram o teor de lectina de soja in natura e processada. Os níveis de lectina mais altos foram encontrados em soja crua (3 $600 \mu \mathrm{g} / \mathrm{g}$ ) e os mais baixos (3,75 a 12,92 $\mu \mathrm{g} / \mathrm{g})$ em produtos de proteína de soja texturizada. Como era esperado, os níveis de atividade de lectina de soja dependeram do processamento térmico.

Aregheore et al. (1998) observaram inativação total de lectinas provenientes de farinha extraída de sementes de Jatropha curcas, quando utilizaram tratamento térmico com calor úmido $\left(66 \%\right.$ de umidade, $121^{\circ} \mathrm{C}$ por 30 minutos) e manutenção da atividade de lectinas com calor seco ( 130 e $160^{\circ} \mathrm{C}$ por 20,40 e 60 minutos) em variedades tóxicas e não tóxicas.

\section{Atividade antinutricional}

As alterações freqüentes, produzidas em animais experimentais após injeção de lectina, são o surgimento de inflamação intensa com destruição das células do epitélio, edema, hiperemia, hemorragia em tecidos linfáticos, degeneração gordurosa e necrose do fígado e lesões do miocárdio e sistema vascular (Jaffé, 1969).

Jaffé \& Brücher (1972) pesquisaram a toxicidade intraperitoneal e oral de lectinas de feijões crus (Phaseolus vulgaris) em ratos. Quatro tipos de lectina foram encontradas nos feijões, embora somente dois tipos tenham apresentado efeitos tóxicos. Ratos que receberam injeções intraperitoneais de extratos de lectinas tóxicas morreram e os animais alimentados com dietas contendo lectinas tóxicas apresentaram perda de peso, crescimento reduzido, baixa absorção de nitrogênio, alterações do baço e pâncreas e após duas semanas morreram.

A toxicidade e inibição do crescimento em animais alimentados com feijões crus, pode ser devida em parte ao efeito das lectinas. Entretanto, deve-se enfatizar que nem todas as lectinas são necessariamente tóxicas ou possuem efeito inibitório sobre o crescimento de animais (Liener, 1974; 1976).

Pusztai \& Palmer (1977) sugeriram que o princípio tóxico do feijão (Phaseolus vulgaris) é idêntico a uma glicoproteína lectina e descartaram a toxicidade de outras proteínas, incluindo o inibidor de tripsina. Por outro lado, Turner \& Liener (1975) pesquisaram em ratos, o efeito da lectina no valor nutricional da soja e sugeriram que, provavelmente, a lectina da soja é o fator que menos afeta o aproveitamento da soja crua como alimento.

Freed \& Buckley (1978) estudaram o efeito da aplicação intragástrica de $50 \mathrm{mg}$ de concanavalina A em ratos. Os exames histológicos demonstraram hipersecreção das células mucosas com subseqüente extravasamento de muco no jejuno dos animais.

Os estudos de Essner et al. (1978) com seis tipos de lectinas fluorescentes, em cólon de ratos, mostraram que as lectinas reagem primariamente com três componentes da mucosa intestinal: muco da cripta luminal, citoplasma e superfície das células absortivas.

Abbey et al. (1979a) sugeriram a existência de outros fatores, tais como as fitohemaglutininas, além do inibidor de protease em feijão-fava cru (Vicia faba L.), como prováveis desencadeadores de efeitos nocivos em ratos. Porém, Lima etal. (1980) estudando as propriedades hemaglutinantes, mitogênicas e tóxicas de 16 variedades de feijão (Phaseolus sp) encontraram que, destas variedades somente 5 apresentaram toxicidade intraperitoneal em ratos, com elevada capacidade aglutinante de hemácias de bovino e coelho e alta atividade mitogênica.

Pusztai et al. (1979) e King et al. (1982) observaram severo rompimento dos microvilos na bordadura em escova localizada nos enterócitos do intestino, em ratos alimentados com dieta contendo lectina de feijão (Phaseolus vulgaris).

Em animais, efeitos tóxicos de lectinas após ingestão oral, podem ser devido a habilidade destas substâncias em 
ligar-se a sítios receptores específicos na superfície das células intestinais, acarretando interferência não específica na absorção de nutrientes (Liener, 1981). Entretanto, o mecanismo do efeito antinutricional das lectinas não é completamente entendido (Sgarbieri \& Whitaker, 1982).

As alterações da função fisiológica causada por lectinas no intestino, parece que são produtos da sua estabilidade aos processos digestivos e a especificidade pelas células da mucosa intestinal em diferentes regiões (Brady et al., 1978; Nakata \& Kimura, 1985). Contudo, não há evidências de que as lectinas presentes nos alimentos apropriadamente processados sejam tóxicas ao homem (Deshpande, 1992).

Os possíveis efeitos adversos de lectinas em humanos podem ser inferidos somente de experimentos com animais de laboratório. Sob esse aspecto, as alterações observadas no intestino e outros órgãos de camundongos, ratos e porcos demonstraram que as lectinas são capazes de provocar reações específicas importantes sob o aspecto de segurança alimentar (Peumans \& Van Damme, 1996). Para Nevel et al. (1998) o fato das lectinas reconhecerem e ligarem-se a receptores glicosilados de células epiteliais localizadas nas vilosidades da mucosa intestinal, conferem a estas proteínas propriedades negativas quanto a interferência nos processos de digestão, absorção e utilização de nutrientes.

\section{CONCLUSÃO}

As leguminosas são fontes importantes de energia e proteína para a dieta em algumas regiões do mundo, portanto a toxicidade de compostos como os inibidores de proteases e lectinas são de considerável interesse, principalmente onde as leguminosas fazem parte do hábito alimentar, uma vez que o valor nutritivo destes alimentos pode ser limitado pela presença de fatores antinutricionais.

A função de inibidores de proteases encontrados em grãos ainda não é completamente entendida, entretanto o potencial de interação fisiológica entre inibidores de proteases de plantas e proteases endógenas de animais não deve ser negligenciado quando considerada suas ações de inibição enzimática.

As lectinas de plantas apresentam diferentes propriedades hemaglutinantes quando testadas com eritrócitos de diversos animais. Para os seres humanos algumas lectinas apresentam toxicidade interagindo com eritrócitos ou apresentando especificidade para determinadas células, enquanto outras não apresentam qualquer reação adversa. As lectinas também despertam interesse científico devido ao grande potencial, quando utilizadas de forma purificada, em pesquisas biológicas para fins de diagnóstico clínico e investigação de estrutura de proteína e carboidratos em células.
Considerando-se a toxicidade dos fatores antinutricionais presentes em alimentos destinados ao consumo humano, em particular, inibidores de proteases e lectinas, deve ser ressaltado que a maior parte destas substâncias parece ser inativada ou inibida quando são utilizados tratamentos térmicos adequados.

\section{REFERÊNCIAS BIBLIOGRÁFICAS}

ABBEY, B.W., NEALE, R.J. , NORTON, G. Nutritional effects of field bean (Vicia faba L.) proteinase inhibitors fed to rats. British Journal of Nutrition, London, v.41, n.1, p.31-38, 1979a.

ABBEY, B.W., NORTON, G., NEALE, R.J. Effects of dietary proteinase inhibitors from field bean (Vicia faba L.) and field-bean meal on pancreatic function in the rat. British Journal of Nutrition, London, v.41, n.1, p.39-45, 1979 b.

AL-WESALI, M., LAMBERT, N., WELHAM, T., DOMONEY, C. The influence of pea seed trypsin inhibitors on the in vitro digestibility of casein. Journal of the Science of Food and Agriculture, Oxford, v.68, n.4, p.431-437, 1995.

ANTUNES, P.L., SGARBIERI, V.C. Processing effects on the nutritive value of soybean seeds and products. Archivos Latinoamericanos de Nutrición, Caracas, v.27, n.1, p.33-47, 1977.

ANTUNES, P.L., SGARBIERI, V.C. Effect of heat treatment on the toxicity value of dry bean (Phaseolus vulgaris var. Rosinha G2) proteins. Journal of Agricultural and Food Chemistry, Washington DC, v.28, n.5, p.935-938, 1980.

AREGHEORE, E.M., MAKKAR, H.P.S., BECKER, K. Assessment of lectin activity in a toxic and a non-toxic variety of Jatropha curcas latex agglutination and haemagglutination methods and inactivation of lectin by heat treatments. Journal of the Science of Food and Agriculture, Oxford, v.77, n.3, p.349-352, 1998.

ASKAR, A. Faba beans (Vicia faba L.) and their role in the human diet. Food and Nutrition Bulletin, Tokyo, v.8, n.3, p.15-24, 1986.

BARCA, A.M.C., VÁZQUEZ-MORENO, L., ROBLES-BURGUEÑO, M.R. Active soybean lectin in foods: isolation and quantification. Food Chemistry, Barking, v.39, n.3, p.321-327, 1991.

BATISTA, I.F.C., OLIVA, M.L.V., ARAUJO, M.S., SAMPAIO, M.U., RICHARDSON, M., FRITZ, H., SAMPAIO, C.A.M. Primary structure of a Kunitz-type trypsin inhibitor from enterolobium contortisiliquum seeds. Phytochemistry, Oxford, v.41, n.4, p.1017-1022, 1996.

BELITZ, H., GROSCH, W. Química de los alimentos. Madrid : Acribia, 1988. Cap 16: leguminosas. p.585-598.

BENDER, A.E. Effects on nutritional balance: antinutrients. In: WATSON, D.H. Natural toxicants in food: progress and prospects. London : Ellis Horwood International Publishers, 1987. p.110-124.

BRADY, P.G., VANNIER, A.M., BANWELL, J.G. Identification of the dietary lectin, wheat germ agglutinin, in human intestinal contents. Gastroenterology, Philadelphia, v.75, n.2, p.236-239, 1978.

CHURELLA, H.R., YAO, B.C., THOMSON, W.A.B. Soybean trypsin inhibitor activity of soy infant formulas and its nutritional significance for the rat. Journal of Agricultural and Food Chemistry, Washington DC, v.24, n.2, p.393-397, 1976. 
DESHPANDE, S.S., NIELSEN, S.S. In vitro digestibility of dry bean (Phaseolus vulgaris L.) proteins: the role of heat-stable protease inhibitors. Journal of Food Science, Chicago, v.52, n.5, p.1330-1334, 1987.

DESHPANDE, S.S., DAMODARAN, S. Food legumes: chemistry and technology. Advances in Cereal Science and Technology, Manhattan, v.10, p.147-241, 1990.

DESHPANDE, S.S. Food legumes in Human nutrition: a personal perspective. Critical Reviews in Food Science and Nutrition, Boca Raton, v.32, n.4, p.333-363, 1992.

ESSNER, E., SCHREIBER, J., GRIEWSKI, R.A. Localization of carbohydrate components in rat colon with fluoresceinated lectins. Journal of Histochemistry and Cytochemistry, New York, v.26, n.6, p.452-458, 1978.

ETZLER, M.E. Plant lectins: molecular and biological aspects. Annual Review Plant Physiology, Palo Alto, v.36, p.209-234, 1985

FREED, D.L., BUCKLEY, C.H. Mucotractive effect of lectin. Lancet, London, v.18, n.1, p.585-586, 1978.

GRANT, G., WATT, W.B., STEWART, J.C., BARDOCZ, S., PUSZTAI, A. Intestinal and pancreatic responses to dietary soyabean (Glycine max) proteins. Biochemical Society Transactions, Colchester, v.16, n.4, p.610-611, 1988.

HASDAI, A., NITSAN, Z., VOLCANI, R. Growth, digestibility, and enzyme activities in the pancreas and intestines of guinea-pigs fed on raw and heated soya-bean flour. British Journal of Nutrition, London, v.62, n.3, p.529-537, 1989.

HOWARD, I., SAGE, H.J., HORTON, C.B. Communication: studies on the appearance and location of hemagglutinins from a common lentil during the life cycle of the plant. Archive of Biochemistry and Biophysics, New York, v.149, n.1, p.323-326, 1972.

JAFFÉ, W.G. Hemagglutinins. In: LIENER, I.E. Toxics constituents of plant foodstuffs. New York : Academic Press, 1969. p.69-101.

JAFFÉ, W.G., BRÜCHER, O. Toxicidad y especificidad de diferentes fitohemaglutininas de frijoles (Phaseolus vulgaris). Archivos Latinoamericanos de Nutrición, Caracas, v.22, n.2, p.267-281, 1972

KAWAGISHI, H., WASA, T., MURATA, T., USUI, T., KIMURA, A., CHIBA, S. Two N-cetyl-D-galactosamine-specific lectins from Phaeolepiota aurea. Phytochemistry, Oxford, v.41, n.4, p.1013-1016, 1996.

KENNEDY, J.F., PALVA, P.M.G., CORELLA, M.T.S., CAVALCANTI, M.S.M., COELHO, L.C.B.B. Lectins, versatile proteins of recognition: a review. Carbohydrate Polymers, Great Yarmouth, v.26, n.3, p.219-230, 1995.

KING, T.P., PUSZTAI, A., CLARKE, E.M.W. Kidney bean (Phaseolus vulgaris) lectin-induced lesions in rat small intestine. Journal of Comparative Pathology, Liverpool, v.92, n.3, p.357-373, 1982.

KOIDE, T., IKENAKA, T. Studies on soybean trypsin inhibitors: 3. amino-acid sequence of the carboxyl-terminal region and the complete amino-acid sequence of soybean trypsin inhibitor (Kunitz). European Journal of Biochemistry, Berlin, v.32, n.3, p.417-431, 1973.

LIDDLE, R.A., GOLDFINE, I.D., WILLIAMS, J.A. Biossay of plasma cholecystokinin in rats: effects of food, trypsin inhibitor, and alcohol. Gastroenterology, Philadelphia, v.87, n.3, p.542-549, 1984.

LIENER, I.E. Phytohemagglutinins: their nutritional significance. Journal of Agricultural and Food Chemistry, Washington DC, v.22, n.1, p.17-22, 1974.

LIENER, I.E. Phytohemagglutinins (phytolectins). Annual Review Plant Physiology, Palo Alto, v.27, p.291-319, 1976.

LIENER, I.E. The nutritional significance of the plant lectins. In: ORY, R.L. Antinutrients and natural toxicants in foods. Westport: Food \& Nutrition Press, 1981. p.143-157.

LIENER, I.E. Trypsin inhibitors: concern for human nutrition or not? Journal of Nutrition, Bethesda, v.116, n.5, p.920-923, 1986.

LIENER, I.E., GOODALE, R.L., DESHMUKH, A., SATTERBERG, T.L., WARD, G., DIPIETRO, C.M., BANKEY, P.E., BORNER, J.W. Effect of a trypsin inhibitor from soybeans (Bowman-Birk) on the secretory activity of the human pancreas. Gastroenterology, Philadelphia, v.94, n.2, p.419-427, 1988.

LIENER, I.E. Implications of antinutritional components in soybean foods. CRC Critical Reviews in Food Science and Nutrition, Boca Raton, v.34, n.1, p.31-67, 1994.

LIMA, A.L., MANCINI FILHO, J., DOMINGUES, J.B., LAJOLO, F.M. Propriedades hemaglutinantes mitogênica e tóxica de variedades brasileiras de feijões (Phaseolus vulgaris L.). Revista de Farmácia e Bioquímica da Universidade de São Paulo, São Paulo, v.16, n.1/2, p.145-154, 1980.

LIS, H., SHARON, N. The biochemistry of plant lectins (phytohemagglutinins). Annual Review of Biochemistry, Palo Alto, v.42, p.541-574, 1973.

MANCINI FILHO, J., LAJOLO, F.M., VIZEU, D.M. Lectins from red kidney beans: radiation effect on agglutinating and mitogenic activity. Journal of Food Science, Chicago, v.44, n.4, p.1194-1196, 1200, 1979.

MARTIN-CABREJAS, M.A., ESTEBAN, R.M., WALDRON, K.W., MAINA, G., GRANT, G., BARDOCZ, S., PUSZTAI, A. Hard-to-cook phenomenon in beans: changes in antinutrient factors and nitrogenous compounds during storage. Journal of the Science of Food and Agriculture, Oxford, v.69, n.4, p.429-435, 1995.

MUELENAERE, H.J.H. Toxicity and haemagglutinating activity of legumes. Nature, London, v.206, n.22, p.827-828, 1965.

MULIMANI, V.H., PARAMJYOTHI, S. Effect of heat and u.v. on trypsin and chymotrypsin inhibitor activities in redgram (Cajanus cajan L.). Journal of Food Science and Technology, v.30, n.1, p.62-63, 1993.

NAKATA, S., KIMURA, T. Effect of ingested toxic bean lectins on the gastrointestinal tract in the rat. Journal of Nutrition, Bethesda, v.115, n.12, p.1621-1629, 1985.

NEVEL, V.C., RYCKE, H., BEECKMANS, S., WILDE, R., DRIESSCHE, E.V. Inhibitory action of spray dried blood plasma and whole egg powder on lectins in extracts of several legume seeds: a qualitative approach. Journal of the Science of Food and Agriculture, Oxford, v.77, n.3, p.319-326, 1998.

NICOLSON, G.L. The interactions of lectins with animal cell surfaces. International Review of Cytology, New York, v.39, p.89-190, 1974. 
NITSAN, Z., LIENER, I.E. Enzymatic activities in the pancreas, digestive tract and feces of rats fed raw or heated soy flour. Journal of Nutrition, Bethesda, v.106, n.2, p.300-305, 1976.

NOAH, N.D., BENDER, A.E., REAIDI, G.B., GILBERT, R.J. Food poisoning from raw red kidney beans. British Medical Journal, London, v.281, n.19, p.236-237, 1980.

OJIMELUKWE, P.C., ONUOHA, C.C., OBANU, Z.A. Effects of processing and in vitro proteolytic digestion on soybean and yambean hemagglutinins. Plant Foods for Human Nutrition, Dordrecht, v.47, n.4, p.293-299, 1995.

PAK, N., MATELUNA, A., ARAYA, H. Efecto de diversos tratamientos termicos en el contenido de hemaglutininas y en la calidad proteca del frijol (Phaseolus vulgaris). Archivos Latinoamericanos de Nutrición, Caracas, v.28, n.2, p.184-195, 1978.

PEUMANS, W.J., VAN DAMME, E.J.M. Prevalence, biological activity and genetic manipulation of lectins in foods. Trends Food Science Technology, Cambridge, v.7, n.4, p.132-138, 1996.

PROLL, J., PETZKE, J., EZEAGU, E.I., METGES, C.C. Low nutritional quality of unconventional tropical crop seeds in rats. Journal of Nutrition, Bethesda, v.128, n.11, p.2014-2022, 1998.

PUSZTAI, A., PALMER, R. Nutritional evaluation of kidney beans (Phaseolus vulgaris): the toxic principle. Journal of the Science of Food and Agriculture, Oxford, v.28, n.7, p. 620-623, 1977.

PUSZTAI, A., CLARKE, E.M.W., KING, T.P., STEWART, J.C. Nutritional evaluation of kidney beans (Phaseolus vulgaris): chemical composition, lectin content and nutritional value of selected cultivars. Journal of the Science of Food and Agriculture, Oxford, v.30, n.9, p.843-848, 1979.

PUSZTAI, A. Lectins. In: CHEEK, P.R. Toxicants of plant origin: proteins and aminoacids. Boca Raton : CRC Press, 1989. v.3: p.29-71.

RACKIS, J.J. Biological and physiological factors in soybeans. Journal of the American Oil Chemist's Society, Champaign, v.51, n.1, p.161A-174A, 1974.

RACKIS, J.J., GUMBMANN, M.R. Protease inhibitors: physiological properties and nutritional significance. In: ORY, R.L. Antinutritional and natural toxicants in foods. Westport : Food \& Nutrition Press, 1982. p.203-237.

SGARBIERI, V.C., WHITAKER, J.R. Physical, chemical, and nutritional properties of common bean (Phaseolus) proteins. Advances in Food Research, New York, v.28, p.93-166, 1982.

SHARON, N., LIS, H. Lectins: cell-agglutinating and sugar-specific proteins. Science, Washington DC, v.177, n.53, p.949-959, 1972.

TURNER, R.H., LIENER, I.E. The effect of the selective removal of hemagglutinins on the nutritive value of soybeans. Journal of Agricultural and Food Chemistry, Washington DC, v.23, n.3, p.484-487, 1975.

XAVIER-FILHO, J., CAMPOS, F.A.P. Proteinase inhibitors. In: CHEEK, P.R. Toxicants of plant origin. Boca Raton : CRC Press, 1989. v.3: p.1-27.

Recebido para publicação em 1 de agosto de 1997 e aceito em 22 de fevereiro de 1999. 